\title{
Suppression of miR-106a-5p expression inhibits tumorigenesis via increasing CELF-2 expression in spinal cord glioma
}

\author{
HAO XU* ${ }^{*}$, FEI WANG ${ }^{*}$ and LIN WANG \\ Department of Neurosurgery, The First Affiliated Hospital of University of Science \\ and Technology of China, Hefei, Anhui 230000, P.R. China
}

Received November 14, 2020; Accepted May 13, 2021

DOI: $10.3892 / \mathrm{ol} .2021 .12888$

\begin{abstract}
Spinal cord glioma is a tumor characterized by high recurrence and mortality rates, and its treatment remains a major challenge. It has been reported that abnormal expression of microRNAs (miRNAs/miRs) is associated with tumor progression. Therefore, the current study aimed to identify novel miRNAs associated with spinal cord glioma. Herein, the expression levels of several miRNAs were determined in human spinal cord glioma and adjacent non-cancerous tissues by reverse transcription-quantitative (RT-qPCR). The results revealed that miR-106a-5p expression was markedly upregulated in spinal cord glioma tissues compared with in non-cancerous tissues. Furthermore, the biological effects of miR-106a-5p on spinal cord glioma cells were evaluated by MTT, Transwell and flow cytometric assays. In 0231SCG cells transfected with miR-106a-5p inhibitor, cell proliferation, migration and invasion were attenuated, whereas apoptosis was enhanced. A search of the TargetScan database revealed that miR-106a-5p directly targeted CUGBP Elav-like family member 2 (CELF-2). Western blot and RT-qPCR experiments further confirmed the association between miR-106a-5p and CELF-2 expression in spinal cord glioma tissues. The current results demonstrated that CELF-2 was a direct target of miR-106a-5p, and that the expression levels of CELF-2 were negatively associated with those of miR-106a-5p. In addition, overexpression of CELF-2 in spinal cord glioma cells reversed the tumor-promoting effects of miR-106a-5p both in vitro and in vivo. Overall, the aforementioned findings indicated that miR-106a-5p, which was highly expressed in spinal cord glioma tissues, may affect the proliferation, migration, invasion and apoptosis of spinal cord glioma cells
\end{abstract}

Correspondence to: Mr. Lin Wang, Department of Neurosurgery, The First Affiliated Hospital of University of Science and Technology of China, 17 Lujiang Road, Luyang, Hefei, Anhui 230000, P.R. China E-mail: tony.neuroscience@gmail.com

*Contributed equally

Key words: spinal cord glioma, tumor progression, microRNA-106a-5p; CUGBP Elav-like family member 2, overexpression via targeting CELF-2, thus indicating a potential approach to the future clinical management of spinal cord glioma.

\section{Introduction}

Spinal cord glioma accounts for $>80 \%$ of all spinal intramedullary tumors $(1,2)$. Due to the high recurrence and poor survival rates of patients with spinal cord glioma, its treatment is considered challenging $(3,4)$. Studies have suggested that the abnormal invasion, proliferation, migration and apoptosis of spinal cord glioma cells are the main obstacles to improving prognosis $(5,6)$. Although progress has been made in conventional treatments, their therapeutic effects are not satisfactory (7). Therefore, the identification of novel therapeutic targets and the development of mechanism-based approaches are urgently required.

MicroRNAs (miRNAs/miRs) are endogenous non-coding single-stranded RNAs 20-25 nucleotides in length, which have regulatory functions and are present in the genomes of most eukaryotes (8). According to the principle of complementary base pairing, the 3'-untranslated region (3'-UTR) of a target mRNA is identified by a miRNA, and the silencing complex is guided to degrade or inhibit the translation of the target mRNA. Certain miRNA precursors can form two stably expressed mature miRNA, with the difference that ' $-3 \mathrm{p}$ ' or ' $-5 \mathrm{p}$ ' is added to the end of the name depending on where it advances (9). Recently, increasing evidence has demonstrated that miRNAs serve an important role in specific cellular processes, such as cell differentiation, morphology and tumor formation $(10,11)$.

During tumorigenesis and tumor development, miRNAs may act as oncogenes or tumor suppressor genes, and are involved in the regulation of tumor-associated genes (12). Studies have suggested that miR-21 (13), miR-10b (14), miR-95-3p (15), miR-106a-5p (16), miR-615-3p (17) and miR-155 (18) are important tumor growth factors. The different expression profiles between glioma and non-cancerous tissues may partially elucidate the roles of different miRNAs in tumorigenesis, and may be therefore used to predict new therapeutic targets (18).

In the present study, the expression levels of miR-21, miR-10b, miR-95-3p, miR-106a-5p, miR-615-3p and miR-155 were compared between spinal cord glioma and non-cancerous spinal cord tissues by reverse transcription-quantitative PCR (RT-qPCR). Since miR-106a-5p was highly expressed in 
spinal cord glioma tissues compared with in non-cancerous tissues, it was hypothesized that miR-106a-5p may serve as a gene mediating the growth of spinal cord glioma. To verify this hypothesis, the effects of miR-106a-5p on cell proliferation, migration, invasion and apoptosis of spinal cord glioma cells were evaluated. Furthermore, the results revealed an inverse association between CUGBP Elav-like family member 2 (CELF-2) and miR-106a-5p expression in human spinal cord glioma samples. Therefore, the effect of CELF-2 overexpression on the biological activity of spinal cord glioma cells was evaluated in vitro and in vivo.

\section{Materials and methods}

Cell culture and transfection. Human spinal cord glioma and non-cancerous spinal cord tissues (normal adjacent tissues) were collected from the Department of Neurosurgery of The First Affiliated Hospital of University of Science and Technology of China (Hefei, China). All 21 patients (admitted between February 2017 and July 2018; age range, 16-60 years; median age, 46 years) provided written informed consent prior to enrolment. The present study was approved by the Institutional Review Board of The First Affiliated Hospital of University of Science and Technology of China. Patient 02-13 was randomly selected for the isolation of spinal cord glioma cells (0213SCG cells). Fresh human spinal cord glioma tissue was cut into $1-\mathrm{mm}^{3}$ pieces and washed 2-3 times with PBS supplemented with $1 \%$ penicillin/streptomycin solution (Gibco; Thermo Fisher Scientific, Inc). PBS was removed and $0.25 \%$ trypsin was added to digest the tumor tissue mass at $37^{\circ} \mathrm{C}$ in a water bath for $30 \mathrm{~min}$. The digested cell fluid was passed through a 100-mesh steel strainer to prepare a tumor cell suspension, which was in turn transferred to a centrifuge tube followed by centrifugation at $241 \mathrm{x} \mathrm{g}$ for $3 \mathrm{~min}$ at room temperature. Subsequently, the cells were resuspended in RPMI-1640 medium (Gibco; Thermo Fisher Scientific, Inc.) containing 10\% FBS (Gibco; Thermo Fisher Scientific, Inc.) and $1 \%$ penicillin/streptomycin solution, and were cultured at $37^{\circ} \mathrm{C}$ in a $5 \% \mathrm{CO}_{2}$ incubator. For tracking transplanted spinal cord glioma cells in vivo, 0213 SCG cells were transduced with a self-inactivating lentiviral vector carrying an ubiquitin promoter driving the expression of firefly luciferase and enhanced green fluorescence protein (Fluc-eGFP) at a multiplicity of infection (MOI) of 10 . After $48 \mathrm{~h}$, fluorescence images of eGFP in $0213 \mathrm{SCG}$ cells were observed at x20 magnification under a fluorescence microscope (Carl Zeiss AG), and luciferase expression was identified. For CELF-2 overexpression in 0213SCG cells, the pcDNA3.1-CELF-2-flag expression vector and blank-vector (pCMV6-entry) were purchased from OriGene Technologies, Inc. 0213SCG cells were transfected with $2 \mu \mathrm{g}$ of the pcDNA3.1-CELF-2-flag plasmid or blank-vector for $24 \mathrm{~h}$ at $37^{\circ} \mathrm{C}$ in serum-free Opti-MEM I (Invitrogen; Thermo Fisher Scientific, Inc.) using Lipofectamine ${ }^{\circledR} 2000$ (Guangzhou RioboBio Co., Ltd.) according to the manufacturer's instructions. Following transfection for $24 \mathrm{~h}$, the culture medium was replaced with fresh complete medium containing $600 \mu \mathrm{g} / \mathrm{ml} \mathrm{G} 418$. The positive 0213SCG cells were obtained after 7 days of screening by G418. Finally, CELF-2 expression was determined by western blot analysis.
Virus particles production. For the production of virus, $293 \mathrm{~T}$ cells (American Type Culture Collection) were seeded into 6-well culture plates. After $24 \mathrm{~h}, 5 \mu \mathrm{g}$ of CMV-eGFP-T2A-Luciferase plasmid (GM-0220IV02; Genomeditech; Jiman Biotechnology (Shanghai) Co., Ltd.), $3 \mu \mathrm{g}$ of package plasmid pCMV.R8.2 and $1 \mu \mathrm{g}$ of envelope plasmid pMD.G were transfected into 293T cells using the standard calcium phosphate (Invitrogen; Thermo Fisher Scientific, Inc.) method (19). The transfected cells were incubated in a $37^{\circ} \mathrm{C}$ incubator with $5 \% \mathrm{CO}_{2}$ for $12 \mathrm{~h}$ and then the medium was replaced with fresh medium. After $48 \mathrm{~h}$ from transfection, virus-containing medium was harvested and centrifuged at $845 \mathrm{x}$ g for $5 \mathrm{~min}$ at room temperature. The virus-containing medium was ultracentrifuged at $50,000 \mathrm{xg}$ for $150 \mathrm{~min}$ at $4^{\circ} \mathrm{C}$ to obtain the high-titer concentrated virus particles.

Cell transfection with miRNA inhibitors/mimics. 0213SCG cells were transfected with $20 \mathrm{nM}$ Homo sapiens miR-106a-5p inhibitors, miR-106a-5p mimics or miRNA negative controls (NCs) using Lipofectamine ${ }^{\circledR} 2000$ (Guangzhou RioboBio Co., Ltd.) for $48 \mathrm{~h}$ at $37^{\circ} \mathrm{C}$ in Opti-MEM (Gibco; Thermo Fisher Scientific,Inc.) using Lipofectamine ${ }^{\circledR} 2000$. Following transfection for $48 \mathrm{~h}$, cells were collected for subsequent investigation. Additionally, the TargetScan database (www.targetscan.org) was utilized to predict the potential target mRNAs of miR-106a-5p. The sequences of all oligonucleotides used for transfection were as follows: Mimics-control sense, 5'-UUC UCCGAACGUGUCACGUTT-3' and antisense, 5'-ACGUGA CACGUUCGGAGAATT-3'; miR-mimics sense, 5'-AAAAGU GCUUACAGUGCAGGUAG-3' and antisense, 5'-ACCUGC ACUGUAAGCACUUUUUU-3'; inhibitor-control, 5'-CAG UACUUUUGUGUAGUACAA-3'; and miR-inhibitor, 5'-CUA CCUGCACUGUAAGCACUUUU-3'.

Cell proliferation assay. For the cell proliferation assay, 0213SCG cells were seeded into 96-well plates at a density of $8 \times 10^{3}$ cells/well. To detect the effects of miR-106a-5p inhibitors on cell proliferation, $0213 \mathrm{SCG}$ cells were transfected with miR-106a-5p inhibitors or NC inhibitors, as aforementioned. Cell proliferation was determined after 24 and $48 \mathrm{~h}$ by MTT assay using dimethyl sulfoxide as the solvent according to the manufacturer's instructions (Sigma-Aldrich; Merck KGaA). Furthermore, to evaluate the effect of CELF-2 overexpression on 0213SCG cell proliferation, 0213SCG cells and 0213SCG cells overexpressing CELF-2 were seeded into 96-well plates at a density of $8 \times 10^{3}$ cells/well. Cell proliferation was then determined at 24 and $48 \mathrm{~h}$ by MTT assay, as aforementioned. The absorbance at $490 \mathrm{~nm}$ was measured to calculate cell proliferation using a multi-function enzyme-linked analyzer (BioTek Instruments, Inc.; Agilent Technologies, Inc.).

Western blot analysis. For CELF-2 protein expression analysis, the cells or tissues were lysed on ice with radio-immunoprecipitation assay buffer supplemented with a protease inhibitor cocktail (Sigma-Aldrich; Merck KGaA). The tumor tissues were specifically lysed for $30 \mathrm{~min}$. BCA reagent (Thermo Fisher Scientific, Inc.) was used to quantify the protein concentration. Total cell or tissue proteins $(50 \mu \mathrm{g} /$ lane) were separated by $10 \%$ SDS-PAGE and transferred onto PVDF membranes (EMD Millipore). 
Following blocking with $5 \%$ skimmed dry milk in Tris-buffer containing 0.05\%-Tween-20 (TBST) for $2 \mathrm{~h}$ at room temperature, the membranes were incubated with primary antibodies at $4^{\circ} \mathrm{C}$ overnight. The membranes were washed thrice with TBST for $5 \mathrm{~min}$ each time, and were then incubated with HRP-conjugated secondary antibodies (1:10,000; cat. no. VA001; Vicmed Biotech Co., Ltd.) at room temperature for $2 \mathrm{~h}$. Following washing with TBST for three times, the blots were visualized using the SuperSignal West Dura Extended Duration Substrate (Thermo Fisher Scientific, Inc). The primary antibodies used were against GAPDH (1:1,000; cat. no. ab9485; Abcam) and CELF-2 (1:400; cat. no. PA1-4130; Thermo Fisher Scientific, Inc.). GAPDH served as the internal control. The grey levels of western blot analysis were measured and quantified using ImageJ software (version ImageJ2; National Institutes of Health).

Tumor model and bioluminescence imaging (BLI). Male nu/nu Nude mice (age, 6-8 weeks; weight 22 g; $n=40$ ) were purchased from the Beijing Weitonglihua Experimental Animal Co., Ltd., and were housed under standard laboratory conditions (temperature, $20-26^{\circ} \mathrm{C}$; relative humidity, 40-60\%; noise, $<60 \mathrm{~dB}$; ventilation degree, 10-20 times/h; light/dark cycle, 12/12 h; adequate food and drinking water.). All experimental procedures were conducted in accordance with the institutional guidelines of the University of Science and Technology of China for The Care and Use of Laboratory Animals and conformed to the National Institutes of Health Guide for Care and Use of Laboratory Animals [approval no. 2019-N (A)-011]. Mice received subcutaneous injection of $100 \mu \mathrm{l}$ of the cell suspension containing $1 \times 10^{6} 0213 \mathrm{SCG}$ cells or CELF-2-overexpressing 0213SCG cells (Fluc-eGFP) into the right forelimb armpit. In addition, the following humane endpoints were established in the present study: i) The tumor burden should not exceed $5 \%$ of the normal body weight of the mouse; ii) the tumor should not grow to a position that seriously affects the normal function of the mouse, and the growth of the tumor should not cause pain in the mouse; iii) the weight loss of mice should not exceed $20 \%$ of the body weight of normal mice; iv) there should be no ulcers or infections at the growth point of the tumor; and v) mice should not exhibit self-harming behavior. Animals were monitored every 3 days to evaluate tumor progression. To monitor tumor development, BLI was applied using the Imaging System IVIS Lumina (Xenogen Corp.). To detect Fluc expression at days 1, 4, 7, 10, 17 and 20, mice were intraperitoneally injected with D-luciferin $(150 \mathrm{mg} / \mathrm{kg}$ body weight; J\&K Scientific Ltd.). The dose of isoflurane used to induce and maintain anesthesia was $2 \%$. For in vitro BLI, 0213SCG cells (Fluc-eGFP) were seeded into 6-well plates at a density of 2, 4, 6 and $8 \times 10^{5}$ cells/well, and D-luciferin was added into each well following incubation for $0.5 \mathrm{~h}$ at room temperature. On day 20 , the mice were euthanized by cervical dislocation, and tumor samples were harvested and fixed with $4 \%$ paraformaldehyde for $48 \mathrm{~h}$ at $4^{\circ} \mathrm{C}$. The largest tumor volume observed in the study was $916 \mathrm{~mm}^{3}$.

Cell migration and invasion assay. Cell migration and invasion assays were performed using Transwell chambers
(8- $\mu \mathrm{m}$ pore; EMD Millipore) pre-coated with (invasion assay) or without (migration assay) Matrigel (cat. no. 356234; Corning, Inc.). Transwell chambers for invasion assays were coated with Matrigel at $37^{\circ} \mathrm{C}$ for $30 \mathrm{~min}$. A total of $1 \times 10^{4}$ 0213SCG cells transfected with miR-106a-5p inhibitors or $\mathrm{NC}$ inhibitors were seeded into the inserts and cultured with serum-free RPMI-1640 medium, and RPMI-1640 medium containing $10 \%$ FBS was added to the lower chambers. Cell migration and invasion were also determined in CELF-2 overexpressing 0213SCG cells. Following culture for $24 \mathrm{~h}$ at $37^{\circ} \mathrm{C}$, non-migrating or non-invading cells on the upper side of the filter were removed with a cotton swab. The migrated or invasive cells were stained with crystal violet for $15 \mathrm{~min}$ at room temperature and counted at 6 randomly selected fields at x20 magnification under an optical microscope (Carl Zeiss AG).

Immunofluorescence staining and TUNEL assay. Tumor tissues formed after transplanting 0213SCG cells were harvested and fixed with $4 \%$ paraformaldehyde for $48 \mathrm{~h}$ at $4^{\circ} \mathrm{C}$ and cut into $5-\mu \mathrm{m}$-thick frozen sections at $-20^{\circ} \mathrm{C}$. The prepared frozen sections were used for immunofluorescence and TUNEL experiments. To evaluate CELF-2 expression in transplanted tumor tissues, immunofluorescence staining was performed using a rabbit anti-human CELF-2 antibody (1:400; cat. no. PA1-4130; Thermo Fisher Scientific, Inc). In addition, apoptosis and proliferation in tumor tissues were detected using the cleaved capase-3 (cat. no. ab49822) and Ki67 (cat. no. ab15580) antibodies (both 1:400; Abcam), respectively. Briefly, the frozen sections were blocked with 5\% BSA (Roche Diagnostics $\mathrm{GmbH}$ ) for $2 \mathrm{~h}$ at room temperature, and then incubated with primary antibody overnight at $4^{\circ} \mathrm{C}$. The primary antibodies were detected by Alexa Fluor 647-labeled secondary antibody (1:1,000; cat. no. A-31573; Invitrogen; Thermo Fisher Scientific, Inc.). Furthermore, the apoptosis in tumor tissues and adherent cells, was evaluated using a TUNEL assay kit (DeadEnd ${ }^{\mathrm{TM}}$ Fluorometric TUNEL System; Promega Corporation) according to the manufacturer's protocol. Tissue sections and apoptotic cells were counterstained with $10 \mu \mathrm{g} / \mathrm{ml}$ DAPI for $20 \mathrm{~min}$ at room temperature and observed at $\mathrm{x} 40$ magnification under a fluorescence microscope (Carl Zeiss AG). The positive area and positive cells were measured using Image-Pro Plus software (version 6.0; Media Cybernetics, Inc.).

Flow cytometric analysis. Flow cytometry was utilized to determine the apoptotic rate of 0213 SCG cells transfected with miR-106a-5p inhibitors and respective NC. Following transfection with miR-106a-5p inhibitors/NCs for $24 \mathrm{~h}, 0213 \mathrm{SCG}$ cells were harvested and stained using an Annexin V and PI kit (BD Bioscience) according to the manufacturer's protocol. Subsequently, the mean fluorescence of the cells was measured and analyzed using the Beckman Coulter FC500 Flow Cytometry system with CXP software (Beckman Coulter, Inc.).

$R T-q P C R$. Total RNA was isolated from tissues or cells using TRIzol ${ }^{\circledR}$ reagent (Invitrogen; Thermo Fisher Scientific, Inc.) according to the manufacturer's protocol. Subsequently, first-strand cDNA was synthesized using reverse transcriptase 

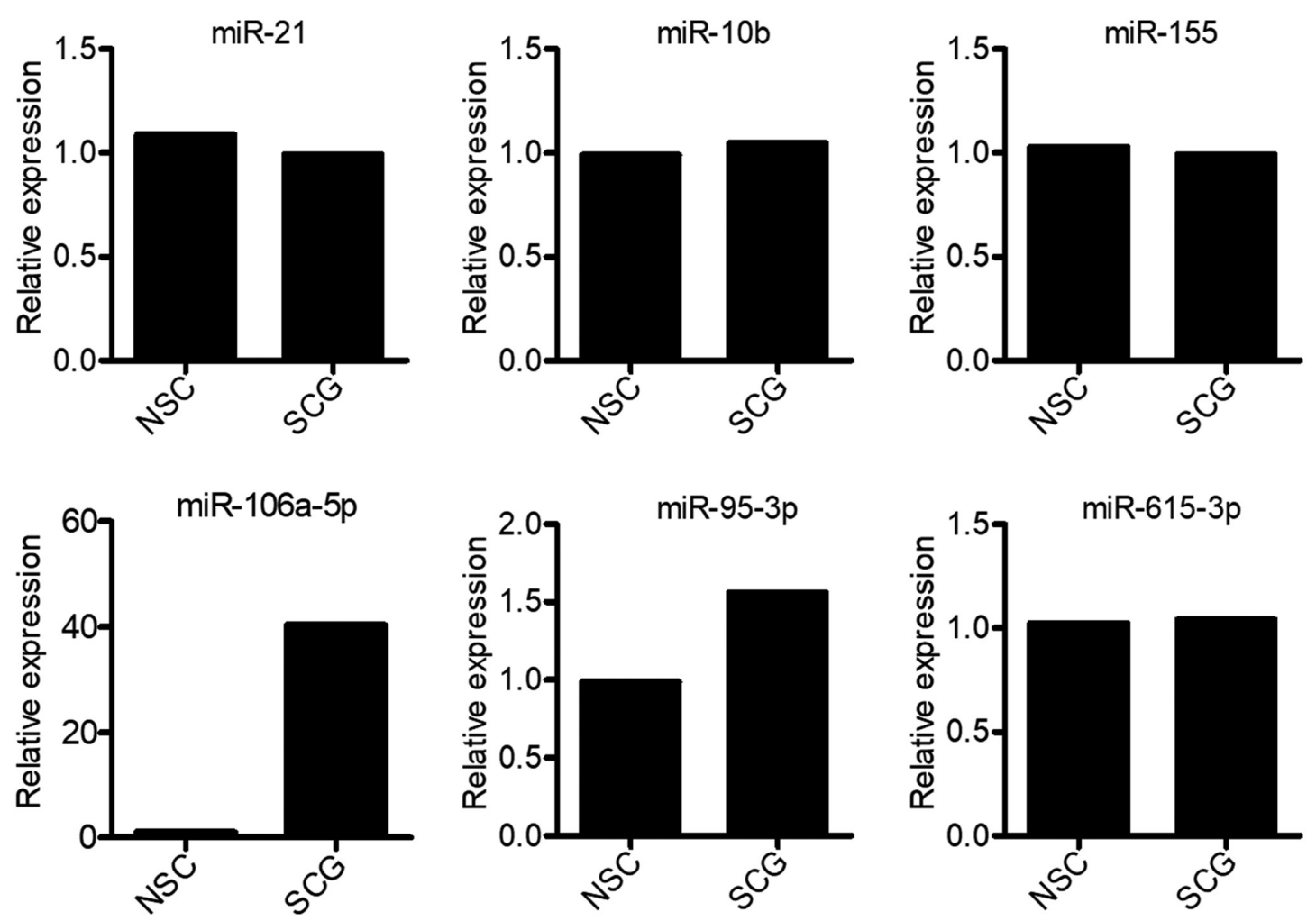

Figure 1. miR-106a-5p expression is significantly upregulated in human SCG tissues from patient 02-13. Reverse transcription-quantitative PCR was used to analyze the expression levels of miR-21, miR-10b, miR-155, miR-106a-5p, miR-95-3p and miR-615-3p in SCG and NSC tissues. All experiments were performed in triplicate. SCG, spinal cord glioma; NSC, normal spinal cord; miR, microRNA.

and oligo(dT) primers of an RT kit (cat. no. AE101-02; TransGen Biotech Co., Ltd.) following the manufacturer's protocol. Real-time PCR was performed with SYBR-Green on the Bio-Rad Real-Time PCR System (Bio-Rad Laboratories, Inc.). The expression levels of miR-21, miR-10b, miR-155, miR-106a-5p, miR-95-3p, miR-615-3p and CELF-2 were quantified using SYBR-Green qPCR (TransGen Biotech Co., Ltd.). The $2^{-\Delta \Delta C q}$ method (20) was used to analyze the relative mRNA fold-changes. Primers are listed in Table SI. In the experiments, GAPDH was used as an internal control for mRNA, and U6 was used as an internal control for miRNA. The thermocycling conditions were as follows: $94^{\circ} \mathrm{C}$ for $5 \mathrm{~min}$, followed by 40 cycles at $94^{\circ} \mathrm{C}$ for $30 \mathrm{sec}, 60^{\circ} \mathrm{C}$ for $25 \mathrm{sec}$ and $72^{\circ} \mathrm{C}$ for $25 \mathrm{sec}$.

Statistical analysis. All statistical analysis was performed using GraphPad Prism 5.0 (GraphPad Software, Inc.). All data were expressed as the mean \pm SEM. Mixed two-way ANOVA was used to evaluate tumor growth at different time points in the control and CELF-2 OE groups, and the Bonferroni test was used as the post-hoc test. Paired Student's t-test was used to analyze the differences between cancerous and non-cancerous tissues. The correlation between Fluc fluorescence intensity and cell number was analyzed by unary linear regression in 0213SCG cells transfected with Fluc-eGFP. For the other datasets, statistically significant differences between two or multiple groups were assessed by unpaired Student's t-test or one-way ANOVA with Tukey's post-hoc test, respectively. $\mathrm{P}<0.05$ was considered to indicate a statistically significant difference.

\section{Results}

miR-106a-5p expression is upregulated in spinal cord glioma tissues. It has been reported that miR-21, miR-10b, miR-95-3p, miR-106a-5p, miR-615-3p and miR-155 are significant regulators of tumor growth (13-18). Therefore, the expression levels of the aforementioned six miRNAs were determined in spinal cord glioma and non-cancerous tissues (normal adjacent tissues) from patient 02-13. The results demonstrated that only miR-106a-5p and miR-95-3p expression was upregulated in spinal cord glioma tissues compared with in non-cancerous tissues (Fig. 1). Compared with normal tissues, miR-106a-5p expression was markedly upregulated in spinal cord glioma tissues, whereas miR-95-3p expression was not markedly altered (Fig. 1). In addition, spinal glioma tissue samples from another 20 patients with high-grade glioma were used as the experimental group, while the non-cancerous spinal cord tissue samples from the corresponding patients served as control. The characteristics of the spinal cord tissue samples are listed in Table SII. The RT-qPCR results indicated that miR-106a-5p was markedly upregulated in spinal glioma tissue samples compared with in normal tissues in all patients (Fig. S1A and B). Therefore, the current findings suggested that miR-615-3p may play a carcinogenic role in spinal cord glioma.

Tumor-promoting effect of miR-106a-5p. Emerging evidence has suggested that miR-106a-5p expression is upregulated or downregulated in different tumor tissues (21-25). However, to the best of our knowledge, the effect of miR-106a-5p in 
A
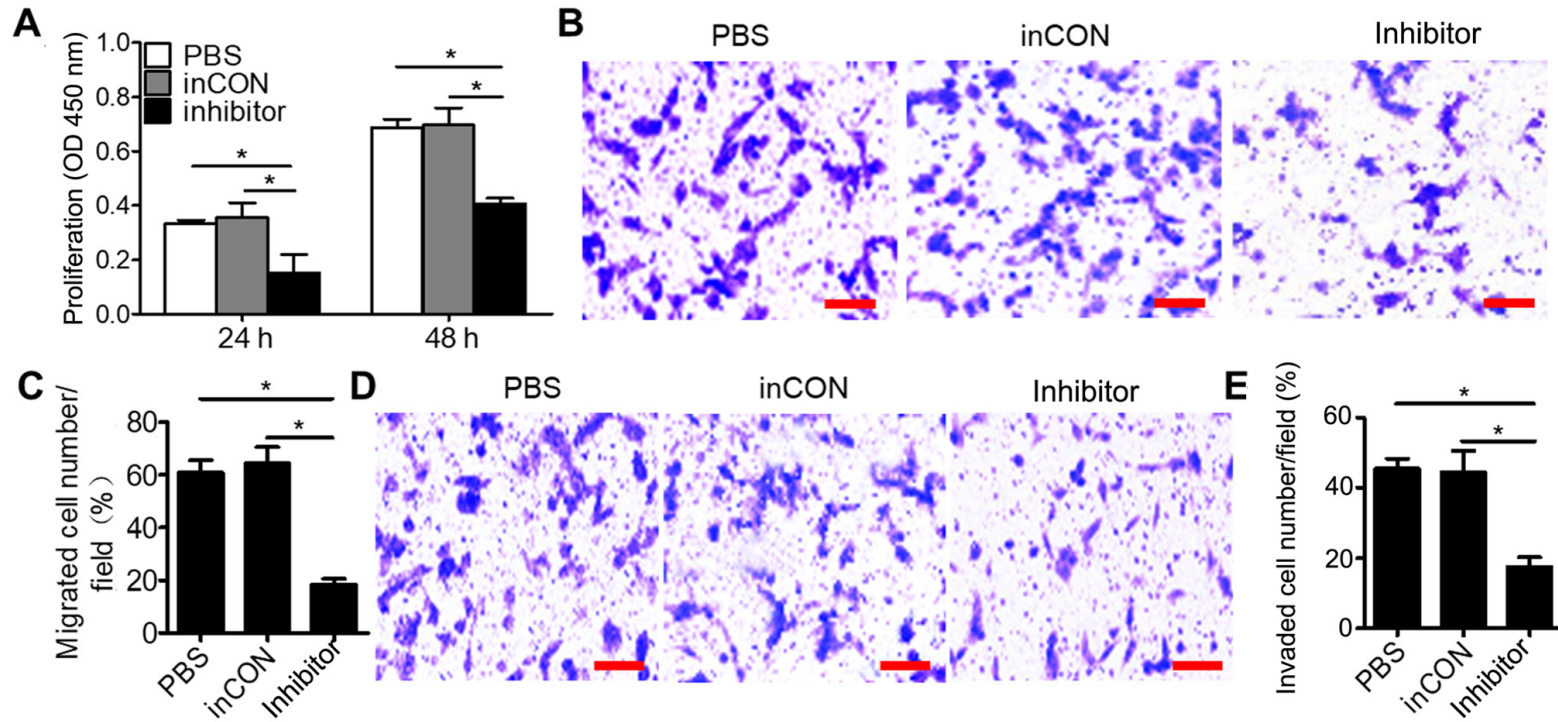

D PBS

BS inCON

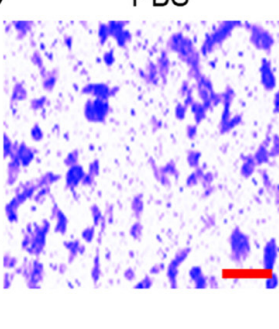

inCON Inhibitor

E

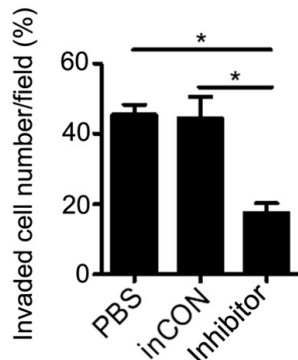

$\mathbf{F}$
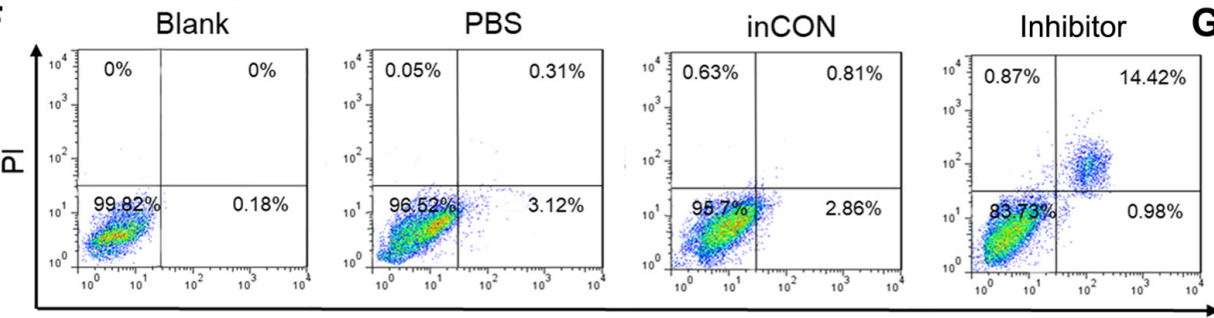

Annexin V

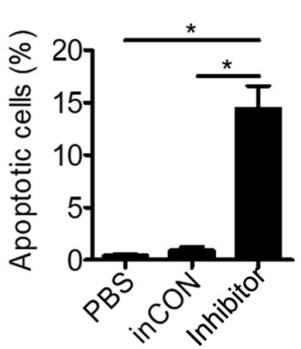

Figure 2. Downregulated miR-106a-5p inhibits the proliferation, migration and invasion, and promotes the apoptosis of 0213SCG cells. (A) MTT assays result indicated that downregulation of miR-106a-5p inhibited the proliferation of 0213SCG cells. (B) Representative photographs of Transwell migration assay of 0213SCG cells treated with PBS, inCON and miR-106a-5p inhibitor. Scale bar, $100 \mu \mathrm{m}$. (C) Quantitative analysis results revealed that the migration of 0213SCG cells was significantly suppressed by the miR-106a-5p inhibitor. (D) Representative photographs of Transwell invasion assay of 0213SCG cells treated with PBS, inCON and miR-106a-5p inhibitor. Scale bar, $100 \mu \mathrm{m}$. (E) Quantitative analysis results revealed that the invasion of $0213 \mathrm{SCG}$ cells was significantly suppressed by the miR-106a-5p inhibitor. (F) Flow cytometry was used to analyze the apoptosis of 0213SCG cells treated with PBS, inCON and miR-106a-5p inhibitor. (G) Quantitative analysis results revealed that miR-106a-5p inhibitors significantly increased the apoptosis of 0213SCG cells. Data are expressed as the mean \pm SEM. ${ }^{*} \mathrm{P}<0.05$. All experiments were performed in triplicate. $0213 \mathrm{SCG}$ cells, spinal cord glioma cells isolated from patient 0213 ; inCON, miR-106a-5p inhibitor negative control; miR, microRNA; OD, optical density.

spinal cord glioma has not been previously investigated. To explore the effect of miR-106a-5p on 0213SCG cells isolated from patients with 02-13 spinal cord glioma, cells were transiently transfected with miR-106a-5p inhibitors or mimics or the corresponding NCs (inCON or miCON, respectively). The expression levels of miR-106a-5p in transiently transfected $0213 \mathrm{SCG}$ cells were determined by RT-qPCR, demonstrating that $0213 \mathrm{SCG}$ cells were efficiently transfected with miR-106a-5p inhibitors or mimics (Fig. S2A and B). Subsequently, cell proliferation was evaluated in 0213SCG cells transfected with miR-106a-5p inhibitors or NC inhibitors for 24 and $48 \mathrm{~h}$ by MTT assays. Therefore, compared with the PBS or inCON groups, the proliferation of 0213SCG cells was significantly attenuated in the miR-106a-5p inhibitor group (Fig. 2A), while the proliferation of 0213SCG cells was significantly increased in the miR-106a-5p mimics group compared with in the PBS and miCON groups (Fig. S2C). Furthermore, cell migration and invasion were evaluated by Transwell assays. Compared with the PBS and inCON groups, $0213 \mathrm{SCG}$ cells transfected miR-106a-5p inhibitors exhibited a significantly attenuated ability to penetrate through the membrane pores pre-coated with or without Matrigel (Fig. 2B-E). Additionally, the apoptosis of 0213SCG cells was analyzed by flow cytometry and TUNEL assay.
The results revealed that $0213 \mathrm{SCG}$ cells transfected with miR-106a-5p inhibitors exhibited a significantly increased apoptosis rate compared with those treated with inCON and PBS (Fig. 2F and G). However, compared with the cells in the miCON and PBS groups, the apoptosis rate was not markedly changed in 0213SCG cells transfected with miR-106a-5p mimics (Fig. S2D). Overall, the aforementioned results suggested that miR-95-3p inhibition affected the proliferation, migration, invasion and apoptosis of $0213 \mathrm{SCG}$ cells.

miR-106a-5p suppresses CELF-2 expression in spinal cord glioma cells. Previous studies have revealed that CELF-2 is a tumor suppressor and is inversely associated with cancer cell migration, invasion, proliferation and apoptosis (26-28). However, the effect of CELF-2 in spinal cord glioma has not been previously reported. To evaluate whether miR-106a-5p could regulate the occurrence of spinal cord glioma via regulating CELF-2, the TargetScan database was utilized to predict the potential target mRNAs of miR-106a-5p. Among all the predicted target-mRNAs, the analysis revealed that CELF-2 could be a putative target gene of miR-106a-5p, since its potential binding site was identified (Figs. 3A and S3). To verify that miR-106a-5p could directly target CELF-2, total RNA and proteins were isolated from transplanted tumor 
A

\begin{tabular}{|c|c|c|c|c|}
\hline & $\begin{array}{l}\text { Predicted consequential pairing of target region (top) } \\
\text { and miRNA (bottom) }\end{array}$ & $\begin{array}{l}\text { Site } \\
\text { type }\end{array}$ & $\begin{array}{l}\text { Context++ } \\
\text { score }\end{array}$ & $\begin{array}{l}\text { Context++ score } \\
\text { percentile }\end{array}$ \\
\hline $\begin{array}{l}\text { Position 5106-5112 of CELF2 3' UTR } \\
\text { hsa-miR-106a-5p }\end{array}$ & $\begin{array}{l}5^{\prime} \ldots \text { UUAGAGGUACUCAGU-CACUUUAA } \ldots \\
\text { III IIIIII } \\
3^{\prime} \quad \text { GAUGGACGUGACAUUCGUGAAA }\end{array}$ & $\begin{array}{c}7 \mathrm{mer}- \\
\mathrm{A} 1\end{array}$ & -0.03 & 54 \\
\hline
\end{tabular}

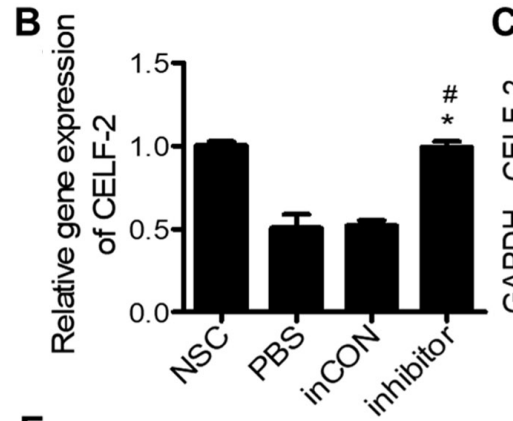

$\mathbf{E}$

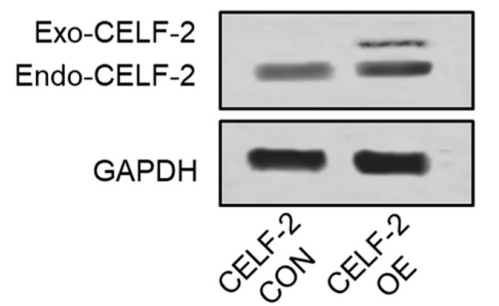

C

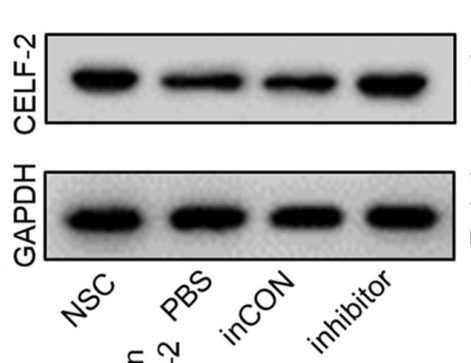

D
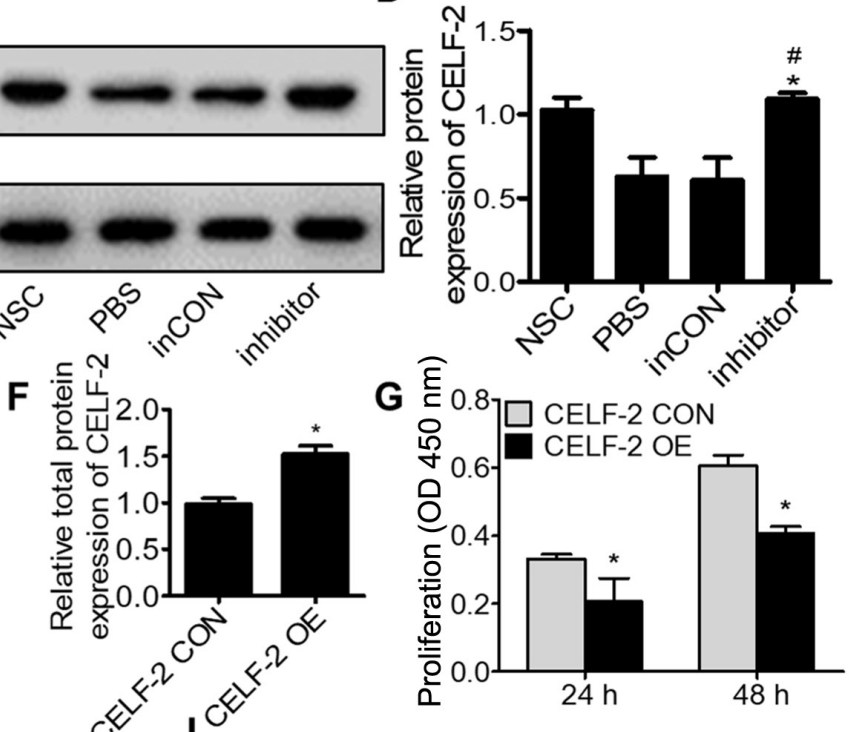

H

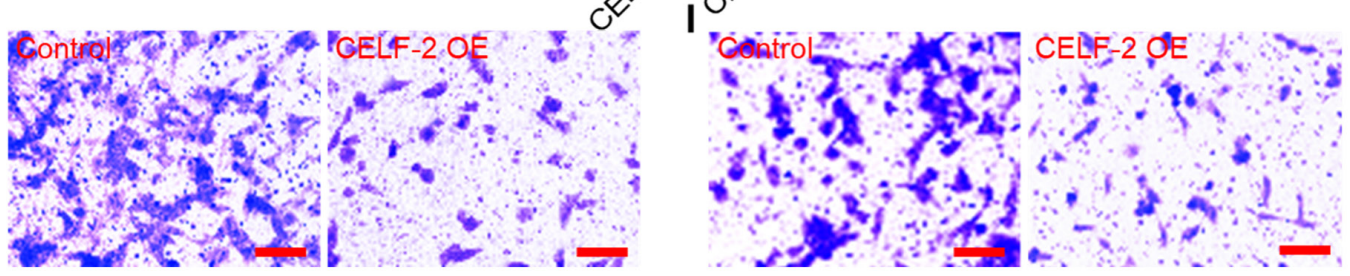

Figure 3. miR-106a-5p promotes tumor progression by inhibiting CELF-2 expression in 0213SCG cells. (A) Predicted binding site of miR-106a-5p in the CELF-2 3'-UTR region using TargetScan. (B) Reverse transcription-quantitative PCR assays showed that the miR-106a-5p inhibitor significantly increased the expression levels of CELF-2 in 0213SCG cells. (C) Western blot results showed the CELF-2 protein expression in 0213SCG cells treated with PBS, inCON and miR-106a-5p inhibitor. (D) Quantitative analysis results revealed that CELF-2 protein expression was significantly increased by the miR-106a-5p inhibitor. Data are expressed as the mean \pm SEM. ${ }^{*} \mathrm{P}<0.05$ vs. PBS; ${ }^{\prime} \mathrm{P}<0.05$ vs. inCON. (E) Western blot analysis of $\mathrm{CELF}-2$ protein expression in CELF-2 OE and CELF-2 CON 0213SCG cells. (F) Quantitative analysis results showed upregulated expression levels of CELF-2 in CELF-2 OE 0213SCG cells. (G) MTT assays results revealed that the overexpression of CELF-2 suppressed 0213SCG cell proliferation at 24 and $48 \mathrm{~h}$. Data are expressed as the mean \pm SEM. ${ }^{*} \mathrm{P}<0.05$ vs. CON. Representative images of Transwell (H) migration and (I) invasion assays in CELF-2 OE and control $0213 \mathrm{SCG}$ cells. Scale bar, $100 \mu \mathrm{m}$. All experiments were performed in triplicate. $0213 \mathrm{SCG}$, spinal cord glioma cells isolated from patient 0213 ; inCON, miR-106a-5p inhibitor negative control; miR, microRNA; CELF-2, CUGBP Elav-like family member 2; OE, overexpression; 3'-UTR, 3'-untranslated region; CON, control; NSC, normal spinal cord tissue adjacent to tumor tissue; OD, optical density; Exo-CELF-2, exogenous CELF-2 derived from the pcDNA3.1-CELF-2-flag plasmid; Endo-CELF-2, endogenous CELF-2.

tissues, 0213SCG cells treated with PBS and 0213SCG cells transfected with miR-106a-5p inhibitors or NC. Subsequently, RT-qPCR and western blot analyses were performed to determine the expression levels of CELF-2. The results revealed that compared with the NSC group, the gene and protein expression levels of CELF-2 were downregulated in the PBS and NC groups; however, cell transfection with miR-106a-5p inhibitors significantly increased the gene and protein expression levels of CELF-2 compared with the PBS and NC groups (Fig. 3B-D).

To determine whether miR-106a-5p promoted the development of spinal cord gliomas via targeting CELF-2, a 0213SCG cell line transiently overexpressing CELF-2 was established. CELF-2 overexpression was verified by western blot analysis (Fig. 3E and F). Subsequently, to reveal whether CELF-2 overexpression could affect the proliferation of $0213 \mathrm{SCG}$ cells, MTT assay was performed. The results indicated that, compared with control $0213 \mathrm{SCG}$ cells, the proliferation of CELF-2-overexpressing 0213SCG cells was significantly attenuated (Fig. 3G). In addition, CELF-2 overexpression markedly decreased the migration and invasion of 0213 SCG cells (Fig. 3H and I). The current findings suggested that CELF-2 may be a functional downstream target of miR-106a-5p regulating the development of spinal cord glioma.

Overexpression of CELF-2 exerts tumor-suppressive effects. To determine the effect of CELF-2 overexpression on spinal cord glioma growth, Fluc imaging was performed to monitor tumor progression. First, to monitor transplanted cells in vivo, an imaging assay was developed using corresponding reporter genes. Transduction of 0213 SCG cells was performed with double fusion (DF) (Fig. 4A). Fluorescence microscopy images 
A

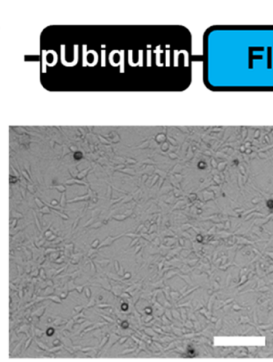

C

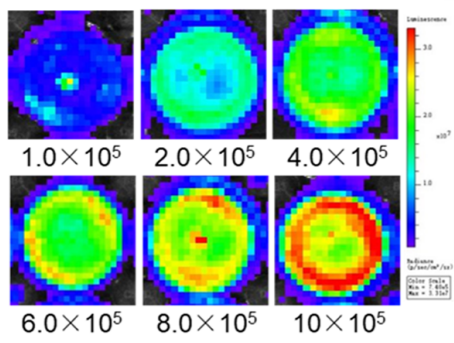

D

E Day1 Day4 Day7 Day10

Day17 Day20
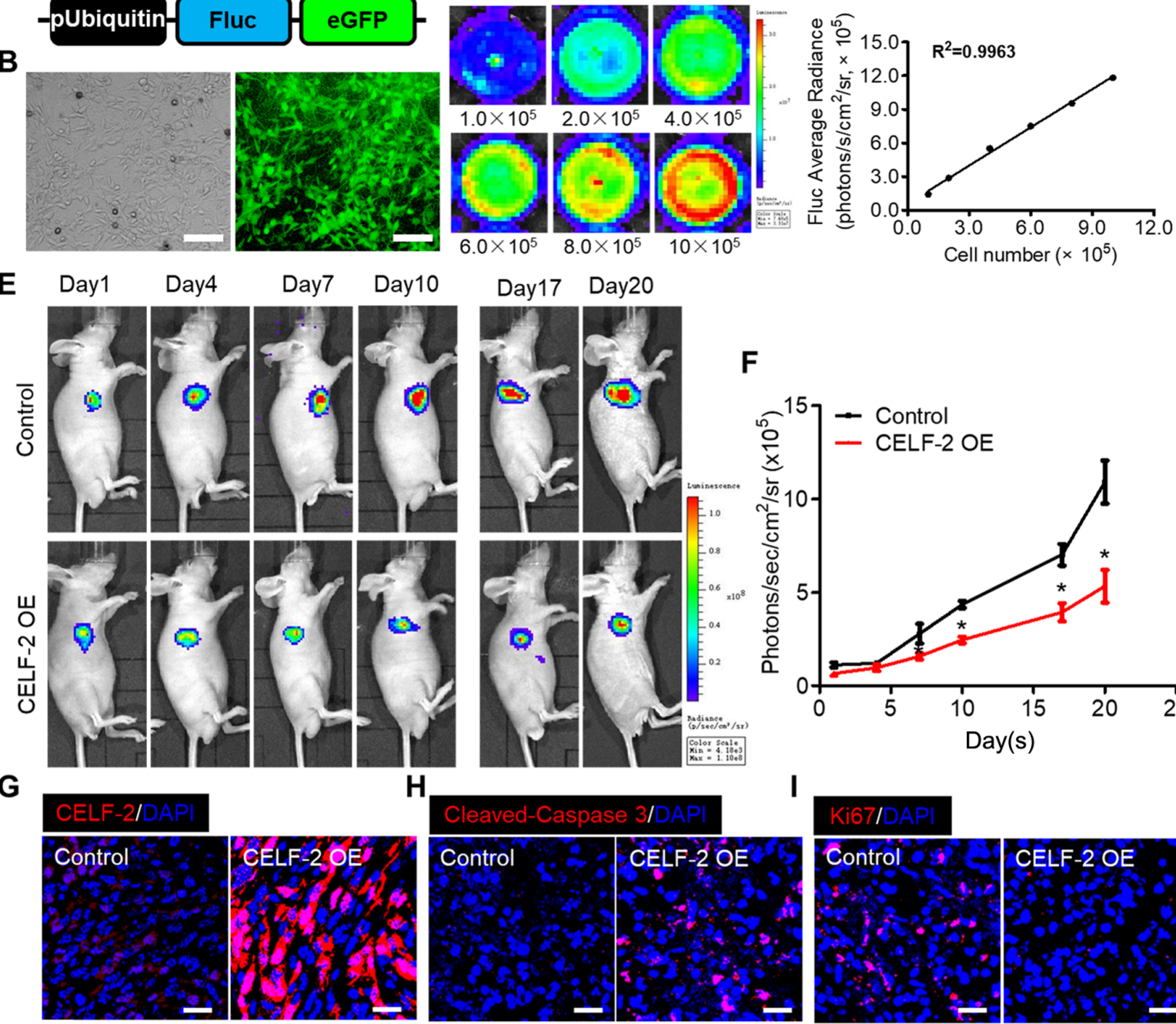

$\mathbf{F}$
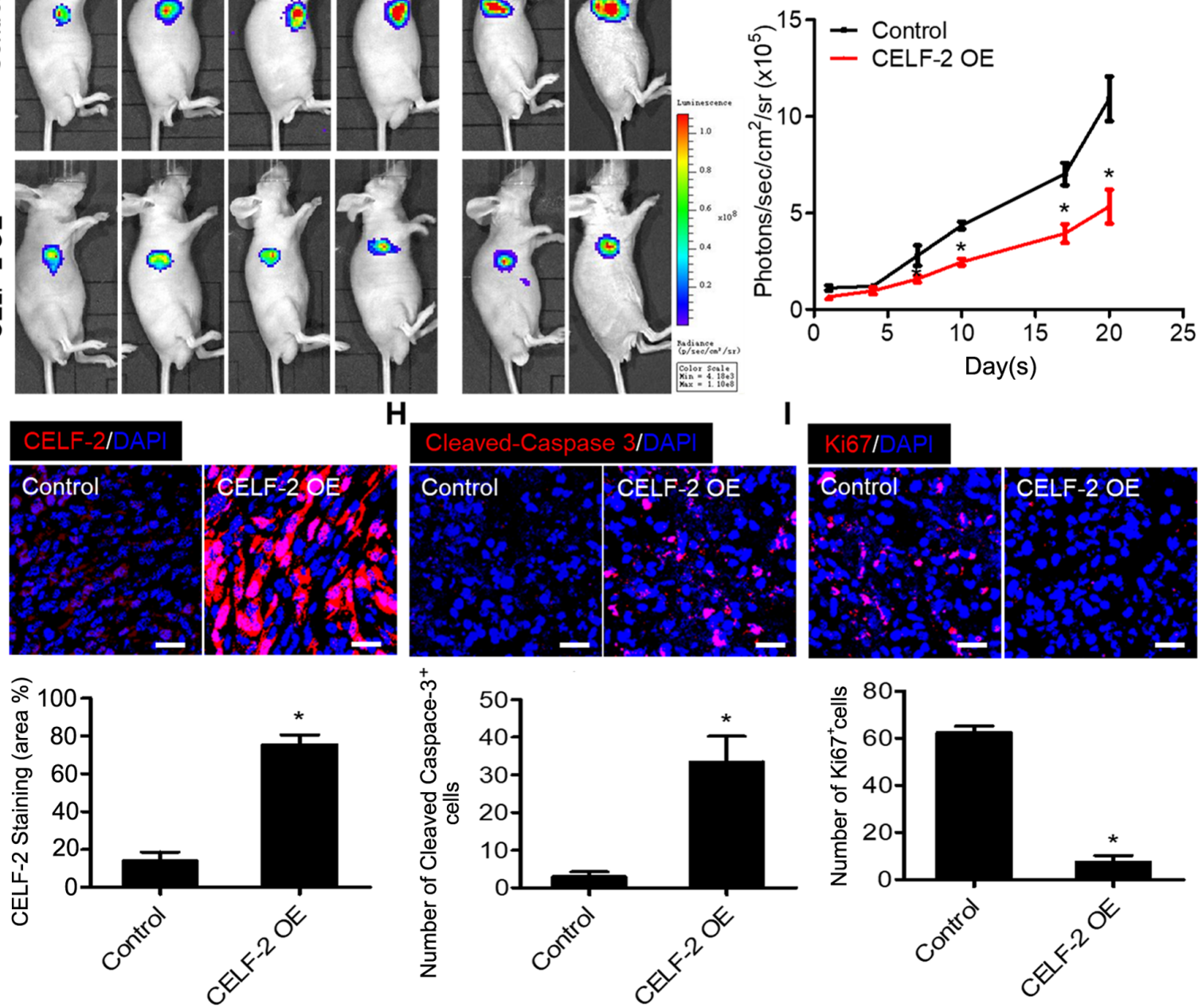

Figure 4. Overexpression of CELF-2 inhibits tumor progression in vivo. (A) Schematic for the double fusion reporter gene construct Fluc-eGFP driven by the ubiquitin promoter. (B) Fluorescence imaging of eGFP in 0213SCG cells transfected with Fluc-eGFP. Scale bar, $100 \mu \mathrm{m}$. (C) Representative bioluminescence images. (D) In vitro imaging analysis of stably transfected 0213SCG cells revealed a strong correlation between cell numbers and Fluc reporter gene activity. (E) Real-time monitoring of tumor progression with Fluc imaging. (F) Quantitative analysis of Fluc signal. (G) Representative images of CELF-2 (red) expression on day 20. Quantitative analysis revealed that CELF-2 protein was highly expressed in the tumors of the CELF-2 overexpression group. Representative images of (H) Cleaved-Capase-3 and (I) Ki67 protein staining (red) on day 20 and quantitative analysis of apoptotic or proliferating cells. Scale bar, $50 \mu \mathrm{m}$. Data are expressed as the mean \pm SEM. "P $<0.05$ vs. Control. All experiments were performed in triplicate. CELF-2, CUGBP Elav-like family member 2; $\mathrm{OE}$, overexpression; eGFP, enhanced green fluorescent protein; Fluc, firefly luciferase.

revealed that eGFP was robustly expressed in 0213SCG cells (Fig. 4B). In addition, a strong association between Fluc activity and 0213SCG cell number was revealed, suggesting that tumor growth may be assessed in vivo by analyzing firefly signal intensity (Fig. 4C and D). A spinal cord glioma model was established in nu/nu nude mice by subcutaneously injecting $1 \times 10^{5} 0213$ SCG cells or CELF-2-overexpressing
0213SCG cells labeled with DF reporter gene into the right forelimb armpit. BLI of Fluc was performed to monitor the development of tumors. Fluc signals were detected at different time points (days 1, 4, 7, 10, 17 and 20). The results indicated that the tumor growth was significantly attenuated in the CELF-2 overexpression group compared with in the control group (Fig. 4E and F). Compared with the control group, 
immunofluorescence staining revealed that CELF-2 was highly expressed in the tumors of the CELF-2 overexpression groups (Fig. 4G). TUNEL staining assay demonstrated a high number of apoptotic CELF-2-overexpressing 0213SCG cells on day 20 (Fig. S4A and B). Furthermore, the results of cleaved-caspase-3 and Ki67 staining indicated that apoptosis was enhanced, whereas cell proliferation was inhibited in the CELF-2-overexpressing 0213SCG cell group (Fig. 4H and I). The aforementioned results suggested that CELF-2 may partially alleviate spinal cord glioma growth.

\section{Discussion}

Several miRNAs have been identified as regulators of multiple biological processes in cancer (29). Herein, the differentially expressed miRNAs (miR-106a-5p, miR-21, miR-10b, miR-155, miR-95-3p and miR-615-3p) were screened in patients with spinal cord glioma by RT-qPCR. The analysis revealed that miR-106a-5p expression was upregulated in spinal cord glioma tissues. Previously studies have suggested that miR-106a-5p may both promote and inhibit tumor progression (21-25). Currently, the effect of miR-106a-5p in spinal cord glioma has not been reported. Therefore, the present study aimed to perform functional experiments on multiple aspects of spinal cord glioma development, including proliferation, migration, invasion and apoptosis. To establish an experimental group, 0213SCG cells isolated from spinal cord glioma tissues were transfected with miR-106a-5p inhibitor to knock down miR-106a-5p expression. MTT assay revealed that transfection with miR-106a-5p inhibitor alleviated the proliferation of 0213SCG cells. Since tumor cell migration and invasion are key factors in tumor metastasis (30), Transwell assays were performed to investigate the effect of miR-106a-5p on these processes. As expected, the number of migrating and invading miR-106a-5p inhibitor-transfected cells was decreased compared with the other groups. Apoptosis analysis revealed that the apoptosis rate was significantly enhanced in the miR-106a-5p inhibitor group.

Subsequently, the current study aimed to uncover the mechanism of miR-106a-5p in spinal cord glioma. The TargetScan online database was used to predict the targets of miR-106a-5p. Among all predicted target mRNAs, CELF-2 was selected as a putative target of miR-106a-5p, since a potential binding site for miR-106a-5p was predicted in the 3'-UTR of CELF-2. Emerging evidence has suggested that CELF-2 acts as a tumor suppressor (26-28). Online miRNA target prediction tools have predicted several potential miRNA targets (www.targetscan.org). However, the regulation of CELF-2 by miRNAs has been poorly studied. It has been reported that miR-95-3p targets CELF-2 in glioma (31). Therefore, miR-95-3p downregulation may inhibit cell proliferation and invasion and promote apoptosis (31). Therefore, the present study hypothesized that CELF-2 may also be associated with the occurrence of spinal cord glioma. RT-qPCR and western blot analysis revealed a negative association between CELF-2 and miR-106a-5p expression in spinal cord glioma tissues. Additionally, CELF-2 may be directly targeted by miR-106a-5p.

A previous study has demonstrated that CELF-2 hypermethylation is associated with shorter overall survival of patients with breast cancer in the clinical setting, while CELF-2 restoration exerted an inhibitory effect on breast cancer growth (32). Therefore, it has been suggested that the epigenetic loss of CELF-2 may enhance breast cancer growth and may be associated with worse outcomes in patients with breast cancer (32). Therefore, the current study hypothesized that CELF-2 may be a potential novel therapeutic target for cancer. Herein, CELF-2 overexpression was shown to attenuate the proliferation, migration and invasion of spinal cord glioma cells in vitro. Furthermore, when CELF-2-overexpressing spinal cord glioma cells were transplanted into mice, BLI revealed that the proliferation of spinal cord glioma cells was alleviated. Additionally, an increased number of apoptotic cells was observed in CELF-2-overexpressing spinal cord glioma tissues. The current findings further supported that CELF-2 may affect the proliferation of spinal cord glioma cells via promoting apoptosis. However, the specific molecular mechanisms underlying the effects of CELF-2 should be further investigated.

Overall, the present study demonstrated that miR-106a-5p expression was abnormally upregulated in spinal cord glioma samples, while miR-106a-5p downregulation inhibited the proliferation, migration and invasion, and promoted apoptosis of spinal cord glioma cells via upregulating CELF-2. In addition, the expression levels of miR-106a-5p and CELF-2 may be associated with the clinical characteristics of patients, so there may be potential applications in clinical practice, although this requires further investigation. In summary, it is important to first find the common characteristics of heterogeneous tumors, and secondly, verify this common point in other samples one by one. Through the systematic analysis of multiple samples, the goal of discovering universal phenomena may be finally achieved.

\section{Acknowledgements}

Not applicable.

\section{Funding}

The present study was supported by the Fundamental Research Funds for the Central Universities (grant no. WK9110000126).

\section{Availability of data and materials}

All data generated or analyzed during this study are included in this published article.

\section{Authors' contributions}

HX and FW performed the experiments and data analysis. HX and LW conceived and designed the study. HX and LW assessed the raw data to ensure its legitimacy. WL reviewed and edited the manuscript. All authors read and approved the final manuscript.

\section{Ethics approval and consent to participate}

The present study was approved by the Ethics Committee of The First Affiliated Hospital of University of Science and 
Technology of China (Hefei, China). Written informed consent was obtained from all patients. The legal guardians of minors or subjects restricted from participating in this study provided informed consent on their behalf. All animal experimental procedures were conducted in accordance with the institutional guidelines of the University of Science and Technology of China for The Care and Use of Laboratory Animals and conformed to the National Institutes of Health Guide for Care and Use of Laboratory Animals [approval no. 2019-N (A)-011].

\section{Patient consent for publication}

Not applicable.

\section{Competing interests}

The authors declare that they have no competing interests.

\section{References}

1. Ma CC, Xiong Z, Zhu GN, Wang C, Zong G, Wang HL, Bian EB and Zhao B: Long non-coding RNA ATB promotes glioma malignancy by negatively regulating miR-200a. J Exp Clin Cancer Res 35: 90, 2016.

2. Ramaswamy V and Taylor MD: CAR T cells for childhood diffuse midline gliomas. Nat Med 24: 534-535, 2018.

3. Tobias A, Ahmed A, Moon KS and Lesniak MS: The art of gene therapy for glioma: A review of the challenging road to the bedside. J Neurol Neurosurg Psychiatry 84: 213-222, 2013

4. Zheng YJ, Liang TS, Wang J, Zhao JY, Yang DK and Liu ZS: Silencing lncRNA LOC101928963 inhibits proliferation and promotes apoptosis in spinal cord glioma cells by binding to PMAIP1. Mol Ther Nucleic Acids 18: 485-495, 2019.

5. Alvi MA, Ida CM, Paolini MA, Kerezoudis P, Meyer J, Barr Fritcher EG, Goncalves S, Meyer FB, Bydon M and Raghunathan A: Spinal cord high-grade infiltrating gliomas in adults: Clinico-pathological and molecular evaluation. Mod Pathol 32: 1236-1243, 2019.

6. Ropper AE, Zeng X, Haragopal H, Anderson JE, Aljuboori Z, Han I, Abd-El-Barr M, Lee HJ, Sidman RL, Snyder EY, et al: Targeted treatment of experimental spinal cord glioma with dual gene-engineered human neural stem cells. Neurosurgery 79 481-491, 2016.

7. An T, Fan T, Zhang XQ, Liu YF, Huang J, Liang C, Lv BH, Wang YQ, Zhao XG, Liu JX, et al: Comparison of alterations in miRNA expression in matched tissue and blood samples during spinal cord glioma progression. Sci Rep 9: 9169, 2019.

8. Gebert LF and MacRae IJ: Regulation of microRNA function in animals. Nat Rev Mol Cell Biol 20: 21-37, 2019.

9. Wessels HH, Lebedeva S, Hirsekorn A, Wurmus R, Akalin A, Mukherjee $\mathrm{N}$ and Ohler U: Global identification of functional microRNA-mRNA interactions in Drosophila. Nat Commun 10: $1626,2019$.

10. Moradimotlagh A, Arefian E, Valojerdi RR, Ghaemi S, Adegani FJ and Soleimani M: MicroRNA-129 inhibits glioma cell growth by targeting CDK4, CDK6, and MDM2. Mol Ther Nucleic Acids 19: 759-764, 2020.

11. $\mathrm{Xu} \mathrm{SJ}, \mathrm{Hu} \mathrm{HT}, \mathrm{Li} \mathrm{HL}$ and Chang S: The role of miRNAs in immune cell development, immune cell activation, and tumor immunity: With a focus on macrophages and natural Killer cells. Cells 8: 1140, 2019.

12. Tang L, Chen HY, Hao NB, Tang B, Guo H, Yong X, Dong H and Yang SM: microRNA inhibitors: Natural and artificial sequestration of microRNA. Cancer Lett 407: 139-147, 2017.

13. Abels ER, Maas SL, Nieland L, Wei Z, Cheah PS, Tai E, Kolsteeg CJ, Dusoswa SA, Ting DT, Hickman S, et al: Glioblastoma-associated microglia reprogramming is mediated by functional transfer of extracellular miR-21. Cell Rep 28 : 3105-3119.e7, 2019.
14. Ouyang H, Gore J, Deitz S and Korc M: Erratum: microRNA-10b enhances pancreatic cancer cell invasion by suppressing TIP30 expression and promoting EGF and TGF- $\beta$ actions. Oncogene 36: 4952-4952, 2017

15. Ye J, Yao Y, Song Q, Li S, Hu Z, Yu Y, Hu C, Da X, Li H, Chen Q and Wang QK: Up-regulation of miR-95-3p in hepatocellular carcinoma promotes tumorigenesis by targeting p21 expression. Sci Rep 6: 34034, 2016.

16. Wang Z, Wang B, Shi Y, Xu C, Xiao HL, Ma LN, Xu SL, Yang L, Wang QL, Dang WQ, et al: Oncogenic miR-20a and miR-106a enhance the invasiveness of human glioma stem cells by directly targeting TIMP-2. Oncogene 34: 1407-1419, 2015.

17. Yan T, Ooi WF, Qamra A, Cheung A, Ma D, Sundaram GM, $\mathrm{Xu} \mathrm{C}$, Xing M, Poon L, Wang J, et al: HoxC5 and miR-615-3p target newly evolved genomic regions to repress hTERT and inhibit tumorigenesis. Nat Commun 9: 100, 2018.

18. Bayraktar R and Van Roosbroeck K: miR-155 in cancer drug resistance and as target for miRNA-based therapeutics. Cancer Metastasis Rev 37: 33-44, 2018.

19. Miyoshi H, Blömer U, Takahashi M, Gage FH and Verma IM: Development of a self-inactivating lentivirus vector. J Virol 72: 8150-8157, 1998.

20. Livak KJ and Schmittgen TD: Analysis of relative gene expression data using real-time quantitative PCR and the 2(-Delta Delta C(T)) method. Methods 25: 402-408, 2001.

21. Luan W, Zhou Z, Ni X, Xia Y, Wang J, Yan Y and Xu B: Long non-coding RNA H19 promotes glucose metabolism and cell growth in malignant melanoma via miR-106a-5p/E2F3 axis. J Cancer Res Clin Oncol 144: 531-542, 2018.

22. He QY, Wang GC, Zhang H, Tong DK, Ding C, Liu K, Ji F, Zhu X and Yang S: miR-106a-5p suppresses the proliferation, migration, and invasion of osteosarcoma cells by targeting HMGA2. DNA Cell Biol 35: 506-520, 2016.

23. Pan YJ, Wei LL, Wu XJ, Huo FC, Mou J and Pei DS: miR-106a-5p inhibits the cell migration and invasion of renal cell carcinoma through targeting PAK5. Cell Death Dis 8: e3155-e3155, 2017.

24. Li D, Wang Z, Chen Z, Lin L, Wang Y, Sailike D, Luo K, Du G, Xiang X and Jiafu GD: MicroRNA-106a-5p facilitates human glioblastoma cell proliferation and invasion by targeting adenomatosis polyposis coli protein. Biochem Biophys Res Commun 481: 245-250, 2016.

25. Hoey C, Ray J, Jeon J, Huang X, Taeb S, Ylanko J, Andrews DW, Boutros PC and Liu SK: miRNA-106a is a novel regulator of radiation resistance through targeting LITAF and ATM in prostate cancer. Mol Oncol 12: 1324-1341, 2018.

26. Pencheva N and Tavazoie SF: Control of metastatic progression by microRNA regulatory networks. Nat Cell Biol 15: 546-554, 2013.

27. Wang J, Liu L, Sun Y, Xue Y, Qu J, Pan S, Li H, Qu H, Wang J and Zhang J: miR-615-3p promotes proliferation and migration and inhibits apoptosis through its potential target CELF2 in gastric cancer. Biomed Pharmacother 101: 406-413, 2018

28. Yeung YT, Fan S, Lu B, Yin S, Yang S, Nie W, Wang M, Zhou L, Li T, Li X, et al: CELF2 suppresses non-small cell lung carcinoma growth by inhibiting the PREX2-PTEN interaction. Carcinogenesis 41: 377-389, 2020.

29. Lin S and Gregory RI: MicroRNA biogenesis pathways in cancer. Nat Rev Cancer 15: 321-333, 2015.

30. Madsen CD, Hooper S, Tozluoglu M, Bruckbauer A, Fletcher G, Erler JT, Bates PA, Thompson B and Sahai E: STRIPAK components determine mode of cancer cell migration and metastasis. Nat Cell Biol 17: 68-80, 2015.

31. Fan B, Jiao BH, Fan FS, Lu SK, Song J, Guo CY, Yang JK and Yang L: Downregulation of miR-95-3p inhibits proliferation, and invasion promoting apoptosis of glioma cells by targeting CELF2. Int J Oncol 47: 1025-1033, 2015.

32. Piqué L, Martinez de Paz A, Piñeyro D, Martínez-Cardús A, Castro de Moura M, Llinàs-Arias P, Setien F, Gomez-Miragaya J, Gonzalez-Suarez E, Sigurdsson S, et al: Epigenetic inactivation of the splicing RNA-binding protein CELF2 in human breast cancer. Oncogene 38: 7106-7112, 2019.

This work is licensed under a Creative Commons Attribution-NonCommercial-NoDerivatives 4.0 International (CC BY-NC-ND 4.0) License. 